\title{
Broad lipid phase transitions in mammalian cell membranes measured by Laurdan fluorescence spectroscopy
}

Nicolas Faerber

University of Augsburg

Christoph Westerhausen ( $\square$ christoph.westerhausen@gmail.com )

University of Augsburg

\section{Research Article}

Keywords: Lipid membrane state, Order disorder phase transitions, Laurdan, Spectral decomposition, Generalized polarization, Cell membrane adaptation

Posted Date: May 13th, 2021

DOl: https://doi.org/10.21203/rs.3.rs-515297/v1

License: (c) (i) This work is licensed under a Creative Commons Attribution 4.0 International License. Read Full License

Version of Record: A version of this preprint was published at Biochimica et Biophysica Acta (BBA) Biomembranes on February 1st, 2022. See the published version at https://doi.org/10.1016/j.bbamem.2021.183794. 


\title{
Broad lipid phase transitions in mammalian cell membranes measured by Laurdan fluorescence spectroscopy
}

\author{
Nicolas Färber ${ }^{1,2}$, and Christoph Westerhausen ${ }^{1,2,3, \sim}$ \\ 1 Experimental Physics I, Institute of Physics, University of Augsburg, Universitätsstr. 1, 86159 Augsburg, Germany \\ 2 Physiology, Institute of Theoretical Medicine, University of Augsburg, Universitätsstraße 2, 86159 Augsburg, Germany \\ 3 Center for NanoScience (CeNS), Ludwig-Maximilians-Universität Munich, 80799 Munich, Germany \\ corresponding author: christoph.westerhausen@gmail.com
}

Employing fluorescence spectroscopy and the membrane-embedded dye Laurdan we experimentally show broad order-disorder-transitions in membranes of various cell lines and red blood cell ghosts. A custom-made setup allows for the determination of fluorescence spectra and the determination of the generalized polarization (GP) as a measure for membrane order in the temperature range of $-40^{\circ} \mathrm{C}$ to $+90^{\circ} \mathrm{C}$ of $\mu \mathrm{l}$-volumes of cell suspension. While artificial lipid membranes like phosphatidylcholine show sharp transitions as known from calorimetry measurements, living cells in a physiological temperature range do only show linear changes in generalized polarization. However, extending the temperature range shows the existence of broad transitions and their sensitivity to cholesterol content, $\mathrm{pH}$ and anaesthetic. Moreover, adaptation to culture conditions like decreased temperature and morphological changes like detachment of adherent cells or dendrite growth are accompanied by changes in membrane order as well. The observed GP changes are equivalent to temperature changes $\mathrm{dT}$ in the range of $-12 \mathrm{~K}<\mathrm{dT}<+6 \mathrm{~K}$.

\section{Keywords}

Lipid membrane state

Order disorder phase transitions

Laurdan

Spectral decomposition

Generalized polarization

Cell membrane adaptation 


\section{Introduction}

Synthetic lipid double layers exhibit cooperative phase transitions causing their physical properties such as heat capacity, volume, area, thickness, stiffness, compressibility, adhesion forces, permeability and diffusion coefficient to depend on temperature, pressure, $\mathrm{pH}$, electric field, salt concentration etc. in a non-linear way [1-9]. This correlation of phase state typically measured by differential scanning calorimetry (DSC) and membrane properties is well investigated for artificially created lipid membranes such as supported lipid bilayers and vesicles. If this concept also holds for biological cell membranes it is not only possible to predict their response to environmental changes but manipulating membrane functions such as permeability becomes possible simply by changing physical parameters like temperature or $\mathrm{pH}$. However, in contrast to their synthetic analogues cell membranes tend to be a cocktail of countless lipid and protein species without sharp transitions and protein addition and lipid mixing lead to transition peak broadening [10,11]. So far Heimburg et al. measured heat capacity profiles of $E$. coli bacteria by DSC and found an about $20 \mathrm{~K}$ broad phase transition some Kelvin below their growth temperature [3]. They could even demonstrate that bacteria cultured at lower temperature actively alter their lipid composition to shift the transition towards lower temperatures suggesting that the membrane phase state is connected to biological functions. However, we were not able to measure transitions in eukaryotic cell membranes by DSC. Reasons are suggested by the data presented below indicating that the transition regime is very broad and extends even below the freezing point of water. Therefore, we measure lipid order optically by analyzing the spectrum of the fluorescent dye Laurdan embedded in the membranes of HeLa, B35 and red blood cell ghosts. Laurdan is sensitive to the polarity of its environment and its fluorescence spectrum is red shifted when surrounded by water molecules due to



Figure 1 Experimental setup and data analysis. a For optical analysis the sample suspension is placed in a glass capillary that can be temperature controlled in a broad temperature range. The fluorescence emission is recorded by a spectrometer attached to an optical fiber. b The membrane embedded dye Laurdan allows for determination of the phase state of lipid membrane samples due to its polarity sensitive emission spectrum. c The fluorescence intensities originating from ordered/disordered lipid domains are determined by data fitting to calculate the generalized polarization as measure for the membrane phase state.

dipolar relaxation [12]. Therefore, its emission maximum shifts from blue (450 $\mathrm{nm}$ ) in the ordered lipid phase with no water present in the bilayer to green $(490 \mathrm{~nm})$ in the disordered phase with water molecules penetrating the membrane. By measuring the blue and green intensity of the Laurdan emission spectrum we calculate the 
Generalized polarization (GP) (Fig. 1c) as a measure of the membrane state [13]. To determine the respective intensities we fit the spectral data by two lognormal functions as proposed by Bacalum et al. [14].

\section{Materials and Methods}

Spectrum acquisition was performed by use of the Ocean optics QEPro spectrometer and an optical fiber connected to the custom-made temperature control setup that is depicted in figure 1a. $600 \mu \mathrm{l}$ of sample suspension containing the membrane of interest were analyzed in the glass capillary embedded in an aluminum block. A stir bar prevented sedimentation and ensured a homogenous temperature distribution within the sample. The temperature was measured with a Pt100 sensor placed in the suspension and regulated in a range from -40 to $95^{\circ} \mathrm{C}$ by a peltier element cooled by a water bath. Laurdan was excited at $360 \mathrm{~nm}$ by an ultraviolet LED with UV-bandpass. The emitted light was captured after an UVband-stop-filter by an optical fiber without any focusing optical elements. Data points were taken in steps of $1^{\circ} \mathrm{C}$. The scan rate was $2^{\circ} \mathrm{C}$ per minute.

Multilamellar phospholipid vesicles were prepared in glass bottles by drying Laurdan (Sigma-Aldrich Chemie GmbH, Munich, Germany) and lipids (Avanti Polar lipids, AL, USA) dissolved in chloroform under nitrogen flow followed by vacuum exposure for at least $60 \mathrm{~min}$. Vesicle swelling in ultrapure water was promoted by ultrasonication $15 \mathrm{~K}$ above the melting transition of the respective lipid species. The final concentration of lipids and Laurdan were $1 \mathrm{mM}$ and $10 \mu \mathrm{M}$.

HeLa cells (ATCC $\AA^{\circ}$ CCL-2 ${ }^{\mathrm{TM}}$ ) and B35 cells (ATCC@ CRL-2754 ${ }^{T M}$ ) were cultured in $25 \mathrm{~cm}^{2}$ NuncTM cell culture flasks (ThermoFisher Scientific, MA, USA) at $37{ }^{\circ} \mathrm{C}$ in saturated atmosphere. The culture media were DMEM for HeLa cells and DMEM with high glucose content (Bio\&SELL GmbH, Nürnberg, Germany) for B35 cells, both supplemented with $10 \%$ fetal bovine serum (FBS Superior) and $1 \%$ Pen/Strep (Biochrom GmbH, Berlin, Germany). Membranes were stained in culture medium after addition of Laurdan dissolved in DMSO for $2 \mathrm{~h}$ at $37^{\circ} \mathrm{C}$. The final concentration of Laurdan and DMSO in the staining solution were $28 \mu \mathrm{M}$ and $141 \mathrm{mM}$ $(11 \mathrm{mg} / \mathrm{l})$. After the staining procedure the cells were rinsed three times with PBS buffer, trypsinated with $1 \mathrm{ml}$ of $0,05 \%$ trypsin/EDTA (Biochrom $\mathrm{GmbH}$, Berlin, Germany) and analyzed without further treatment.

Cholesterol depleted HeLa cells were prepared by addition of $5 \mathrm{mM}$ Methyl-betacyclodextrin after staining and trypsination in the above-described manner. After $1 \mathrm{~h}$ treatment time without stirring at $37^{\circ} \mathrm{C}$ the suspension was centrifuged at $300 \mathrm{~g}$ for 3 min and the supernatant was replaced by fresh PBS buffer. Before optical analysis the cells pellet was resuspended by vortexing. In contrast to the temperature scan the cholesterol depletion kinetics were analyzed under steady stirring.

HeLa cell adaptation was introduced by lowering the culture temperature by $1^{\circ} \mathrm{K}$ per week. After reaching the final temperature of $30^{\circ} \mathrm{C}$ no cell division took place for about one month. Over a period of further 3 month the cell's growth rate increased up to one division per week. After the adaptation phase the cells were analyzed as described above.

B35 cells with octanol were prepared by using a trypsin solution with $1 \mathrm{mM}$ octanol for trypsination after staining. The sample was directly afterwards analyzed.

Differentiated B35 cells were obtained after incubation of a confluently grown cell flask in a $2 \mathrm{mM}$ DcAMP culture medium solution at $37^{\circ} \mathrm{C}$ for $24 \mathrm{~h}$.

Red blood cell ghosts were prepared following the procedure of Himbert et al. [15]: $300 \mu \mathrm{l}$ of venous blood (friendly supplied by University Hospital Augsburg) were diluted with 1700 $\mu$ l PBS buffer followed by centrifugation for $3 \mathrm{~min}$ at $600 \mathrm{~g}$ and subsequent removal of the 
supernatant. After repeating this washing procedure three times the cells were ruptured by exposure to hypotonic buffer (1.6ml PBS buffer and $48.4 \mathrm{ml}$ ultra-pure water buffered with sodium hydroxide at $\mathrm{pH} 8$ ) for 30min in an ice bath. The sample was centrifuged at $18000 \mathrm{~g}$ for $30 \mathrm{~min}$ followed by buffer exchange. The following three washing steps were performed at $18000 \mathrm{~g}$ for $15 \mathrm{~min}$. The ghosts were stained in the hypotonic buffer containing $28 \mu \mathrm{M}$ Laurdan and $141 \mathrm{mM} \mathrm{DMSO}$ for $2 \mathrm{~h}$ at $37^{\circ} \mathrm{C}$. Then the sample was again centrifuged at $18000 \mathrm{~g}$ for $15 \mathrm{~min}$ and the staining solution was replaced by buffer adjusted with monopotassium phosphate and disodium phosphate adjusted to the $\mathrm{pH}$ values 5,6 and 7 . This solution was analyzed after resuspending the ghost by vortexing.

Multilamellar HeLa and B35 membrane vesicles were prepared by following the lipid extraction protocol of Folch et al. [16]. After trypsination about 2 mio cells were centrifuged after trypsination at $300 \mathrm{~g}$ for 3 min followed by supernatant removal. The pellet was transferred into $2 \mathrm{ml}$ of a chloroform/methanol solution (2:1) and lyzed by sonication for $30 \mathrm{~min}$ at $70^{\circ} \mathrm{C}$. After addition of $400 \mu \mathrm{l}$ ultra-pure water and vortexing for $30 \mathrm{sec}$ the solution was centrifuged at $100 \mathrm{~g}$ for $15 \mathrm{~min}$. The lower part of the resulting biphasic solution, containing mainly chloroform and lipids, was transferred into another glass vial and Laurdan dissolved in chloroform was added. After drying under nitrogen flow and vacuum exposure for at least $60 \mathrm{~min}$ the lipids were hydrated with $1 \mathrm{ml}$ of PBS buffer and vesicle swelling was induced by ultrasonication at $70^{\circ} \mathrm{C}$ for $30 \mathrm{~min}$. The final concentration of Laurdan was $10 \mu \mathrm{M}$. The lipid content can be estimated using the findings of Delgado et al. who measured for mouse embryonic fibroblasts that lipids account for 13 percent of 500pg dry mass per cell [17]. If we use these values for our cell lines it leads to 130pg of lipids per sample. With a rough guess for the mean molecular lipid weight of about $500 \mathrm{~g} / \mathrm{mol}$ we estimate the final lipid concentration to be $260 \mu \mathrm{M}$.
Statistics The data points of the shown GP temperature scans of cellular membranes represent the mean value of three independent measurements. The error bars show the standard deviation or in case of the $\triangle \mathrm{GP}$ curves the pooled standard deviations. We performed two tailed t-tests with 4 degrees of freedom to test for $5 \%$ statistical significance between GP values. The fit parameters of the Boltzmann sigmoid function were compared by two tailed t-tests on $5 \%$ statistical significance as well. The data of three independent sample downscans (130 data points per scan from $90^{\circ} \mathrm{C}$ to $-40^{\circ} \mathrm{C}, 390$ points in summary, 4 fit parameters and therefore 386 degrees of freedom) were approximated by one function. The number of degrees of freedom for comparison of two fit parameters were 772 for a scan from $90^{\circ} \mathrm{C}$ to $-40^{\circ} \mathrm{C}$.

\section{Results and Discussion}

\section{Calorimetric and optical analysis of phospholipid vesicles}

First, we examined the possibility of measuring the phase state of synthetic lipid membranes optically in a temperature range from $\mathrm{T}=-40^{\circ} \mathrm{C}$ to $\mathrm{T}=95^{\circ} \mathrm{C}$. Therefore, we compared calorimetric data of five different vesicle suspensions with temperature scans of the GP value and its derivative with respect to temperature (Fig. 2). These measurements were performed in a temperature controlled glass capillary that is schematically shown in figure $1 \mathrm{a}$. Our data proves that we are in case of 13:0PC, 14:0PC, 15:OPC and 15:0PC with $10 \%$ cholesterol not only able to detect the correct location of phase transitions in lipid membranes but also peak width and height show similar behavior in both data sets. According to literature DOPC shows a phase transition as measured by DSC at $\mathrm{T}=-16.5^{\circ} \mathrm{C}, \mathrm{T}=-17.3^{\circ} \mathrm{C}$ and $\mathrm{T}=-21^{\circ} \mathrm{C}[18-$ 20]. Thus, it is suited to examine if there are still Laurdan dynamics in membranes surrounded by frozen water. Figure 2 show that even though we see a drop in the derivate of the GP curve at $\mathrm{T}=0^{\circ} \mathrm{C}$ (Fig. $2 \mathrm{a}$ inset) indicating that the GP value is 

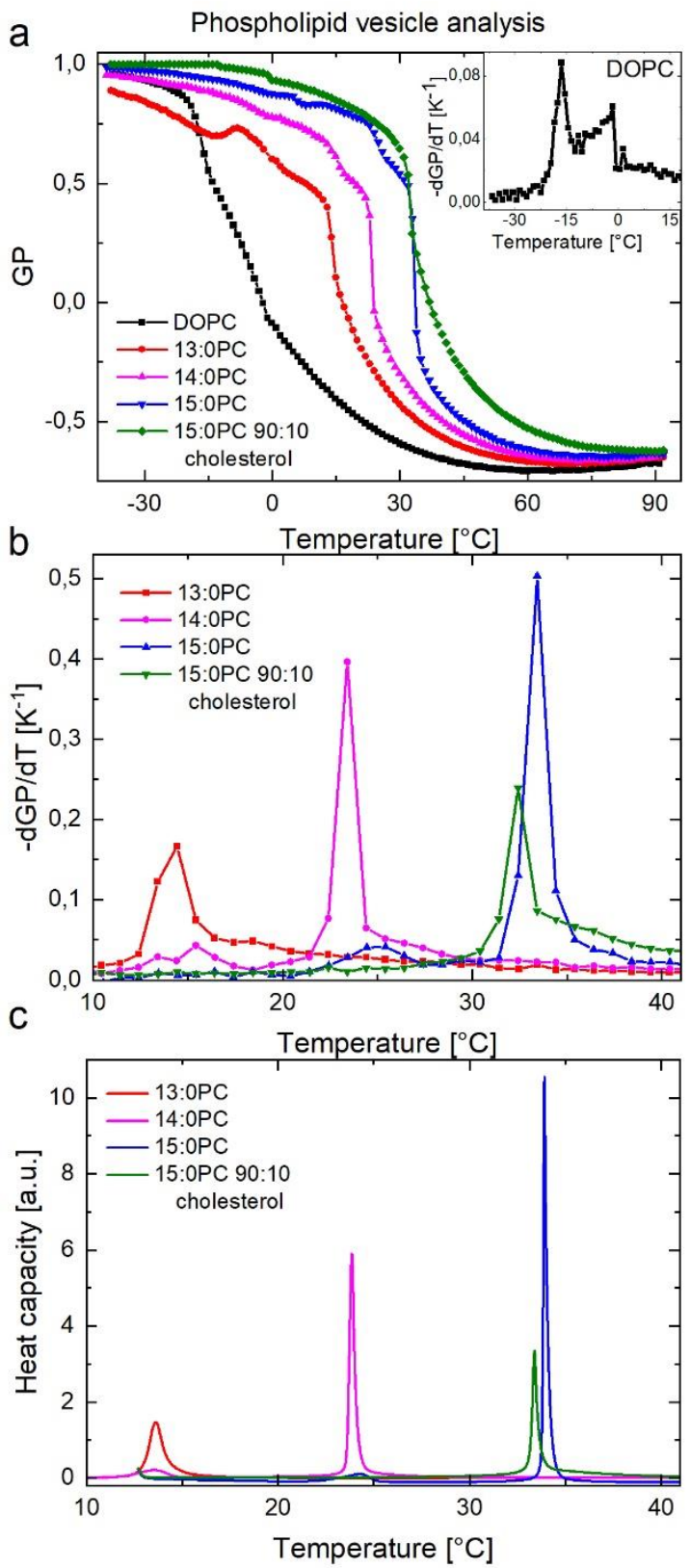

Figure 2 Phospholipid vesicle analysis. a Temperature dependent generalized polarization of various phospholipid multilamellar vesicles measured during heating. The inset shows the derivative with respect to temperature of DOPC vesicles with a peak at $\mathrm{T}=-16.5^{\circ} \mathrm{C}$ indicating the ordered/disordered phase transition. $\mathbf{b}$ Derivative with respect to temperature of various lipid vesicles showing the same ordered/disordered phase transitions as their heat capacity profiles measured by DSC in c.

somehow affected by water freezing it is still possible to detect the phase transition optically since our data shows peaks at $\mathrm{T}=-16.5^{\circ} \mathrm{C}$ in the up-scan and at $\mathrm{T}=-19.4$ ${ }^{\circ} \mathrm{C}$ in the down scan (SI).

HeLa cell membrane state, adaptation and cholesterol depletion
In the following, we compare such phase state measurements on synthetic and biological membranes. For the latter we always analyzed the down scan since the GP changes stay reversible after the first heating (Supplementary Fig. 1a). The deviations between the first upscan and all following scans can be attributed to denaturation processes. Figure 3 shows the temperature dependent Laurdan emission spectra of 14:0PC multilamellar vesicles (MLV) and HeLa cell membranes. In both cases close to $T=25^{\circ} \mathrm{C}$ the emission shifts from blue to green indicating a change from an ordered to a disordered state. The difference lies in the steepness of the transition indicating differences in cooperativity. The peak in the derivative with respect to temperature of the 14:OPC MLV sample is 20 times higher than the one of the HeLa cells. The small peak height meaning low cooperativity might be the reason why we were not able to measure the transition by DSC and may be attributed amongst others to the variability of cell membranes and high cholesterol content in eukaryotic cell membranes. When we investigated phase state changes during the trypsination process of HeLa cells we found a variation of GP values at constant temperature of $\quad \Delta \mathrm{GP} \approx 0.02$ (Supplementary Fig. $1 \mathrm{~b}, \mathrm{c}$ ). During this measurement about 100 cells were in the focus range of the spectrometer. Therefore, one can conclude that the variability of single cells is even higher and the presented temperature scans including millions of cells in suspension are a sum of various slightly different melting events resulting in a broad transition. As shown in figure 2, cholesterol reduces the cooperativity in synthetic membranes. This and the fact that bacteria are not able to synthesize cholesterol [21] might be the reason why we could not identify phase transitions on HeLa cells using DSC.

To examine the effect of cholesterol content on the phase state of HeLa membranes we depleted the membranes by exposure to methyl-beta-cyclodextrin. Figure 4 shows GP as function of temperature for wild type, 

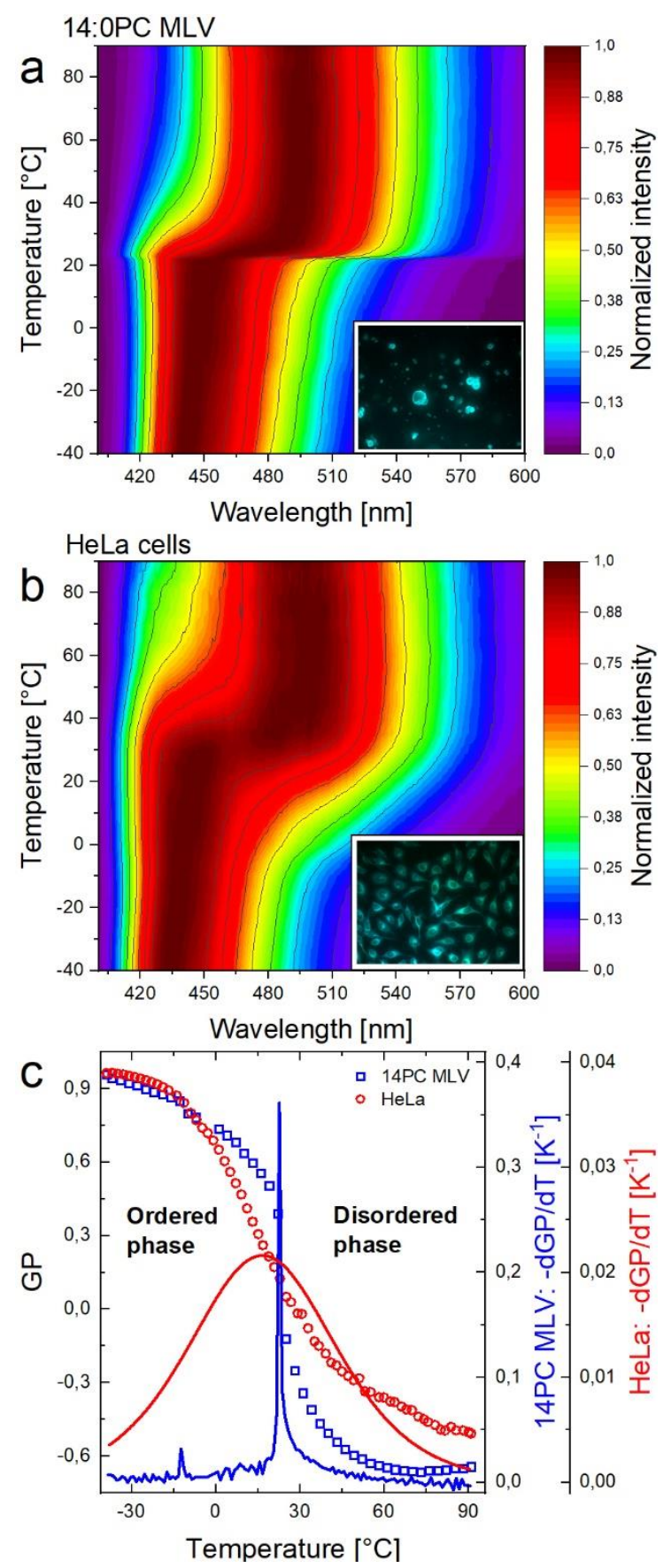

Figure 3 Comparison of phospholipid vesicles and HeLa cell membranes. $\mathbf{a}, \mathbf{b}$ Normalized emission spectra of 14:OPC multilamellar vesicles/HeLa cells as a function of temperature and fluoresccnce microscopy images of the samples (field of view about $420 \times 320 \mu \mathrm{m}^{2}$ ). c GP of 14:OPC multilamellar vesicles/HeLa cells as a function of temperature and their corresponding derivatives after temperature at two differently scaled axes.

growth temperature adapted, cholesterol depleted HeLa cells and lipid extracts from these cells. Figure $4 a$ shows that cholesterol depletion caused a shift of the GP curve especially at around $T=30^{\circ} \mathrm{C}$ towards lower values meaning increased disorder in the membrane. Consequently,

the transition becomes steeper and the cooperativity determined by the steepness of a Boltzmann sigmoid fit function at the inflection point increases by $23 \%$, as shown in figure $4 \mathrm{~b}$. The other fit parameters namely the span and inflection point corresponding to melting enthalpy $\Delta \mathrm{H}$ and melting Temperature $\mathrm{T}_{\mathrm{m}}$ are affected as well and decreased by $\Delta \mathrm{H} / \mathrm{H} \approx-4 \%$ and $\Delta \mathrm{T}_{\mathrm{m}}$ $\approx-2.4 \mathrm{~K}$. The inset in Figure $4 \mathrm{a}$ shows the GP values in a physiological temperature regime. A linear approximation of GP as function of temperature for the reference HeLa cells in a temperature regime from $20^{\circ} \mathrm{C}$ to $40^{\circ} \mathrm{C}$ gives a slope of $5.3 \mathrm{~K}$ per 0.1 GP. Using this slope, we can convert GP changes into equivalent temperature changes $\Delta \mathrm{T}$, as this might be more intuitive than bare changes in membrane (dis)order. Figure 4c shows these equivalent temperature changes $\Delta \mathrm{T}$. Following this concept, the cholesterol depletion induces a decrease in lipid order that corresponds to an increase in temperature of $\Delta \mathrm{T}=+7 \mathrm{~K}$ to $\Delta \mathrm{T}=+11 \mathrm{~K}$ (Fig. 4c).

Heimburg et al. measured phase transitions in the lysate of Escherichia coli bacteria which contain much less cholesterol in their membranes by DSC. They found a transition slightly below the culture temperature. Furthermore, this transition shifted towards lower temperatures after culture below physiological temperatures [22]. Inspired by these findings we decreased the culture temperature of $\mathrm{HeLa}$ cells from $T=37^{\circ} \mathrm{C}$ to $T=30^{\circ} \mathrm{C}$ (temperature ramp $-1 \mathrm{~K} /$ week) and kept it at $T=30^{\circ} \mathrm{C}$ over a period of about 5 months before we measured membrane order as function of temperature (Fig. $4 \mathrm{a}, " 30^{\circ} \mathrm{C}$ culture"). In accordance with bacteria data the GP values of these eukaryotic cells decreased by $\Delta \mathrm{T}=2.5 \mathrm{~K}$ temperature equivalent (Fig. 4c) due to adaptation meaning that the cells compensate the increased order caused by the lower temperature by adjusting the membrane composition to reduce order again. The position of the derivative peak is almost not affected but a $3 \%$ increase of slope $\mathrm{C}$ as measure for the cooperativity is seen (Fig. 4b). This could indicate a change 

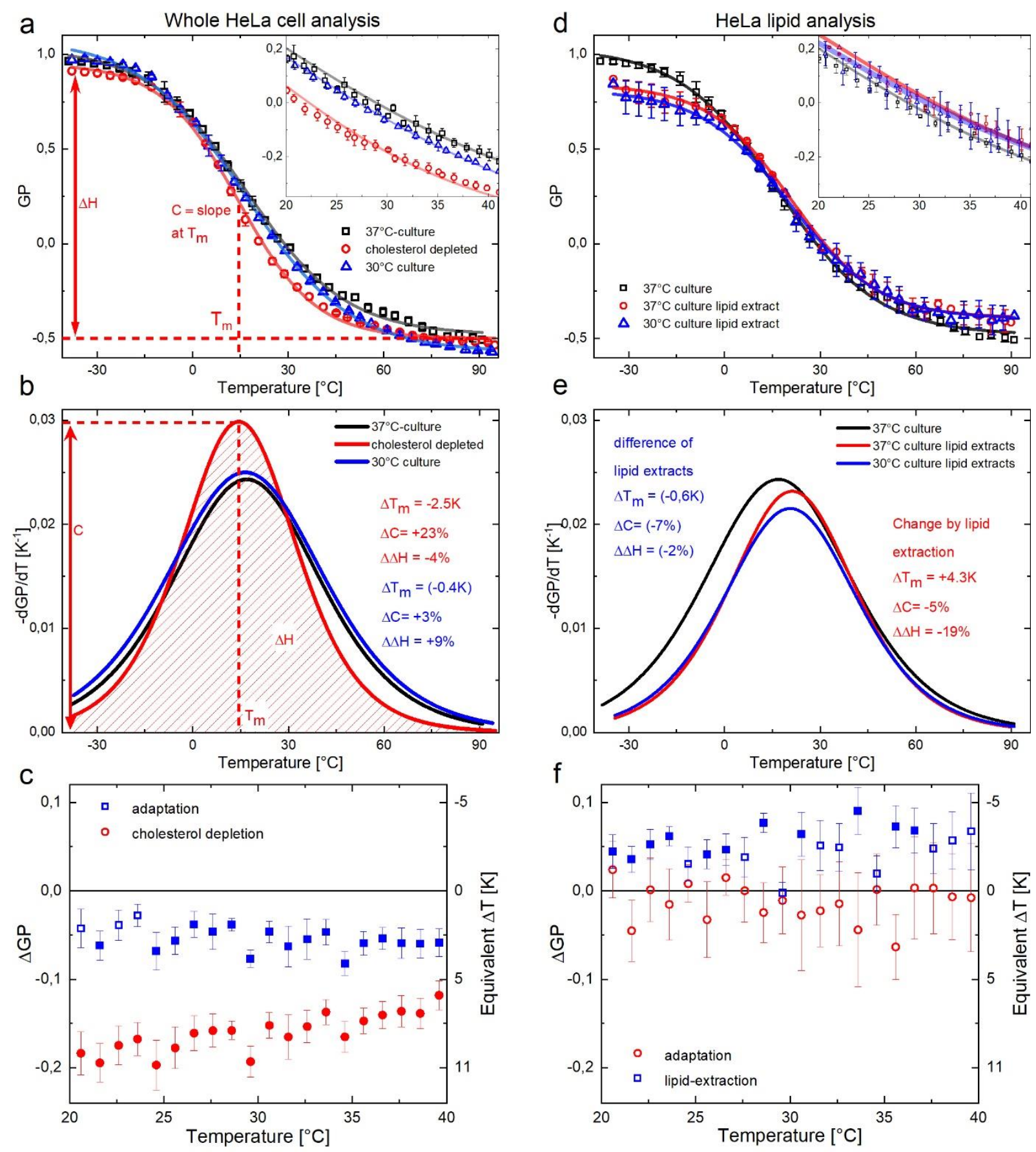

Figure 4 Phase state analysis of HeLa cell membranes and their lipid extracts under varying conditions. a, $d$ Temperature dependent GP values of untreated, cholesterol depleted and low temperature $\left(30^{\circ} \mathrm{C}\right.$ ) adapted HeLa cell membranes and their lipid extracts. Each data point represents the mean value with standard deviation of at least 3 independent samples. For visibility only every fourth point is shown. The data is approximated by a Boltzmann sigmoid function which allows for determination of the point of inflection or $\mathrm{Tm}$, its steepness as a measure for cooperativity $\mathrm{C}$ and the span corresponding to a melting enthalpy $\Delta \mathrm{H}$. The inset shows GP values in a physiologically relevant temperature interval and the $95 \%$ confidence intervals of the according fit functions. b,e Derivatives after temperature of GP graphs pictured in A and D. The changes of melting temperature $\Delta \mathrm{Tm}$, cooperativity $\Delta \mathrm{C}$ and melting enthalpy $\Delta \Delta \mathrm{H}$ due to adaptation, cholesterol depletion and lipid extraction are calculated by comparison of the Boltzmann sigmoid fit parameters. c,f Temperature dependent GP changes induced by adaptation, cholesterol depletion and lipid extraction. Data points with a P-value larger than $5 \%$ are represented by hollow symbols. The equivalent temperature changes are calculated by the use of a linear approximation of untreated HeLa cells in the temperature regime from $20^{\circ} \mathrm{C}$ to $40^{\circ} \mathrm{C}$.

of cholesterol content during adaptation. To examine whether the GP change is caused by alteration of lipids or protein we applied the Folch method to split a cell lysate into a water soluble part containing most of the proteins and an unpolar part with all the 
lipid molecules of the cells [16]. The latter was dried and rehydrated to obtain HeLa cell membrane multilamellar vesicles. The comparison of the temperature dependent GP values of regular HeLa cells with their lipid extracts and lipid extracts of adapted HeLa cells is shown in figure $4 d$. The lipid extract transition differs strongly in melting temperature, cooperativity and enthalpy from the transition of the intact HeLa cell samples not only because of the lack of proteins but in this experiment lipids of all cell organelles are probed, not only the outer membrane. The comparison of the lipid extracts of reference and adapted cells again show that adaptation decreases lipid order, but the effect is weaker, especially in the physiological temperature range. Figure $4 \mathrm{f}$ shows that no $\Delta \mathrm{GP}$ value differs from 0 with a $p$ value lower than 0.05 but a trend towards negative $\Delta \mathrm{GP}$ values as observed for the whole cells is visible. The derivative of GP with respect to temperature of the adapted cells show a peak shifted for $\Delta T=-0.6 \mathrm{~K}$ towards lower temperatures. In contrast to adaptation processes measured in figure $4 b$ the cooperativity and melting enthalpy of the adapted HeLa cell lipids are lower than the reference lipid extract.

The lipid extracts contain lipids from the whole lipid membrane reservoir in the cell interacting with only few proteins, while the intact cell analysis only probes the outer membrane interacting with the membrane proteins. This can explain why the adaptation effect is decreased in lipid extracts and clearly show the advantage of analyzing intact cells. Since both samples show differences between adapted and reference cells, we conclude that the lipid composition indeed is altered but we cannot exclude adaptation of protein expression as well. However, following the idea of determination of phase diagrams of intact cells to correlate these with cellular function somehow move this detail out of focus.

\section{Neuroblastoma cell membrane after differentiation and anaesthetic exposure}

To contribute to the discussion whether phase transitions in biological membranes play a role in the signaling mechanism of nerve cells $[3,23,24]$ we analyzed B35 neuroblastoma cells and how their membrane order-disorder-transition is affected by differentiation and exposure to an anaesthetic. Applying the same concept as shown in figure 4 for HeLa cells, figure 5 shows GP as function of temperature, it's derivative with respect to temperature in absence and presence of octanol and differentiated as well as undifferentiated. It is known for lipid vesicles that the phase transition temperature is lowered after incorporation of an anaesthetic meaning that the disordered phase expands [25]. In figure 5a we see that the same applies for B35 Neuroblastoma membranes when they get exposed to $1 \mathrm{mM}$ octanol. We see a GP change equivalent to about $\Delta \mathrm{T}=-2 \mathrm{~K}$ to $\Delta \mathrm{T}$ $=-5 \mathrm{~K}$ left shift in the physiological regime (Fig. 3c) and a global peak shift of $\Delta \mathrm{T}=-3 \mathrm{~K}$ (Fig. 3b). While the melting enthalpy of the transition is almost not affected by octanol addition, the cooperativity is decreased by $3 \%$. The effect of anaesthetic exposure was examined by the use of undifferentiated B35 cells, which do not form neurites. We treated these cells for $24 \mathrm{~h}$ with Dibutyryladenosine 3',5'-cyclic monophosphate sodium salt (DcAMP) to promote neurite outgrowth and examined the phase state of these differentiated cells. The measured GP change after differentiation corresponds to a shift of up to $\Delta \mathrm{T}=-4 \mathrm{~K}$ towards lower temperatures (Fig. 5c) accompanied by a movement of the melting transition by $\Delta T_{m}=-1.4 \mathrm{~K}$ in the same direction. Furthermore, the melting enthalpy and cooperativity are reduced by differentiation (Fig. 5b). We performed measurements on the lipid extracts of differentiated and undifferentiated B35 cells and find again by comparison of whole cell analysis and lipid extracts that the transition of the lipid extracts becomes more narrow (Fig. 4e and 5e). However, comparing lipid 

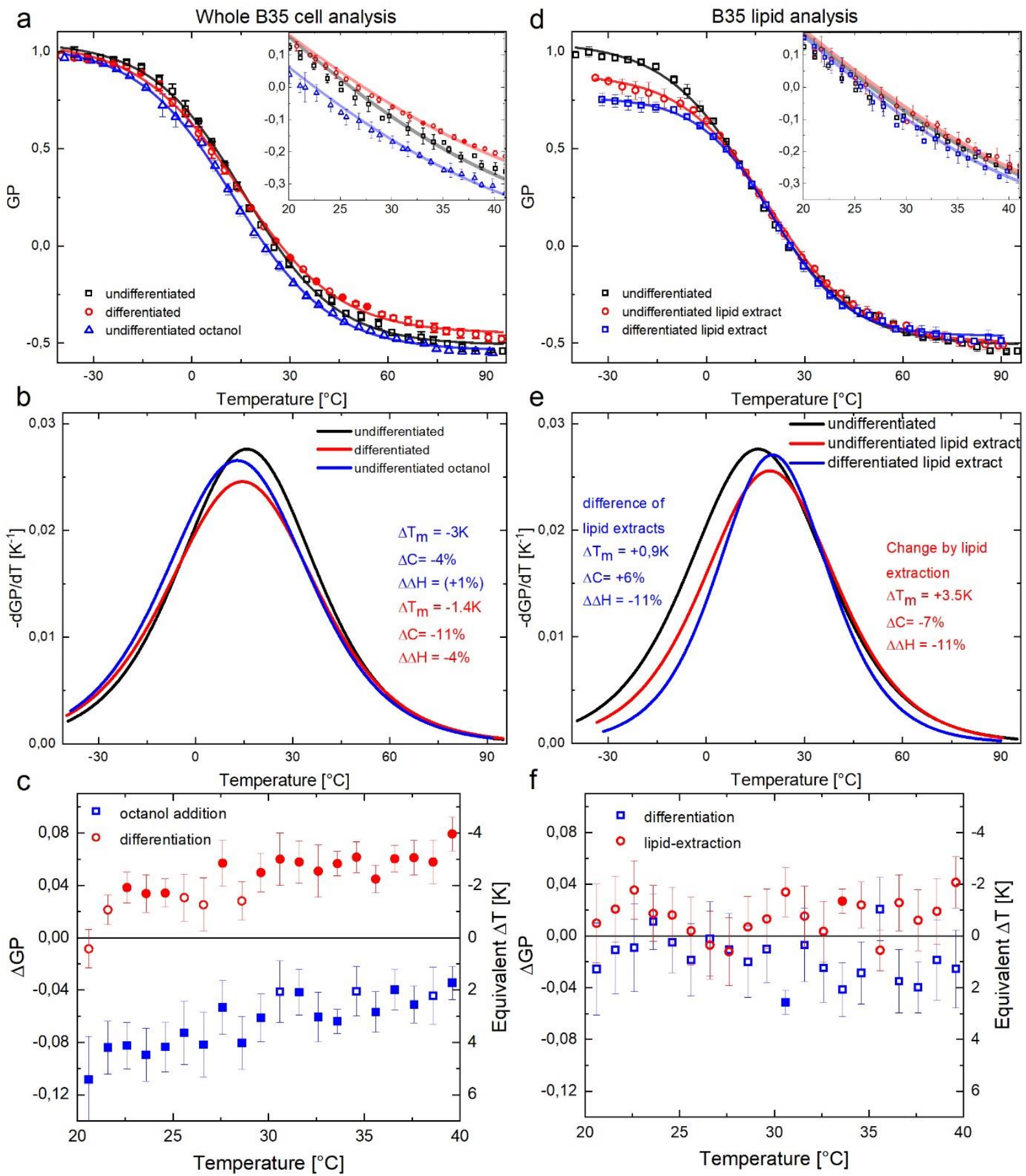

Figure 5 Phase state analysis of B35 Neuroblastoma cell membranes and their lipid extracts under varying conditions. a,d Temperature dependent GP values of untreated, differentiated and octanol ( $1 \mathrm{mM})$ exposed cell membranes and their lipid extracts. Data point representation, error bars and fit values in a-f are treated as described in figure 4.

extracts of differentiated and undifferentiated cells reveal a small positive shift $\Delta T$ in contrast to whole cells. This could indicate that the lipid composition of cell organelles is altered in a different way upon differentiation than the outer membrane. The latter is for example more affected by increased membrane tension due to outgrowth of microtubuli than inner membranes.

\section{Red blood cell ghosts at different $\mathrm{pH}$ levels}

To stress the multidimensionality of membrane phase diagrams, in the following we analyze order disorder transitions in red blood cells that are sensitive for $\mathrm{pH}$ changes. It is known that red blood cells (RBC) show protonation dependent membrane properties e.g. permeability [26] that can be understood by charge effects in 


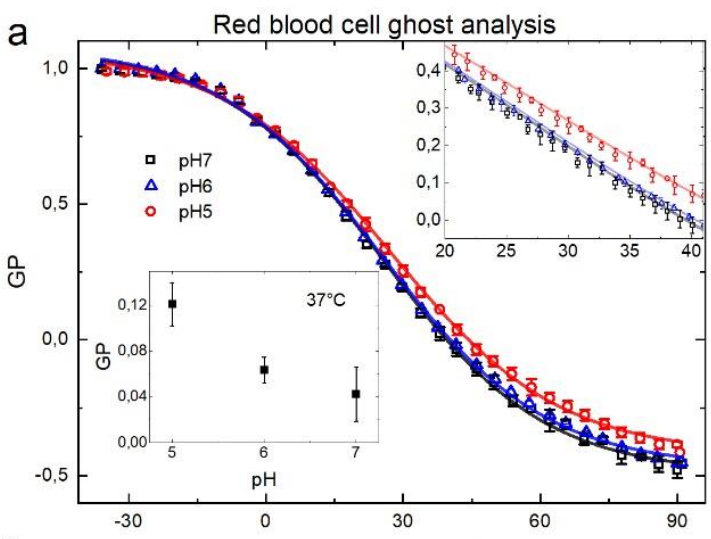

b

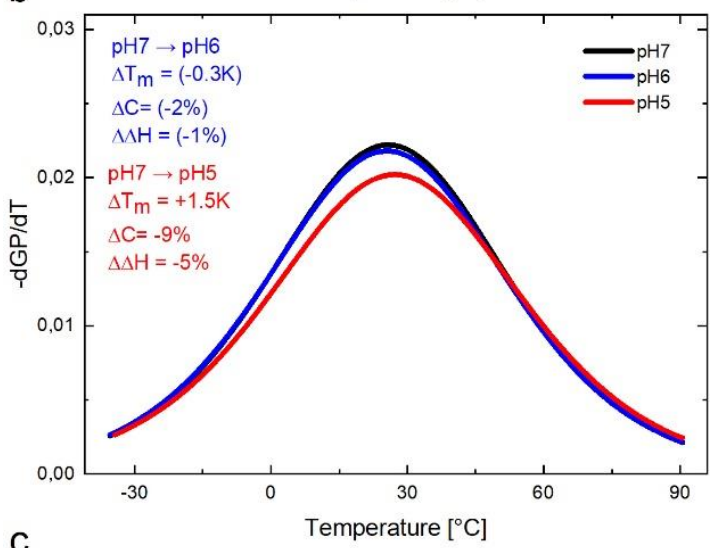

C

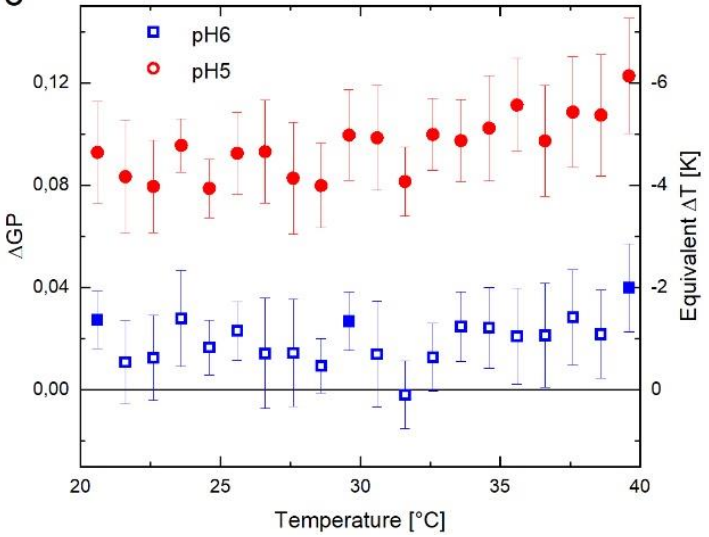

Figure 6 Phase state analysis of red blood cell ghosts under varying $\mathrm{pH}$ levels. a Temperature dependent GP values of red blood cell ghosts at pH 7, pH 6 and pH 5 . Data point representation, error bars and fit values in a-c are treated as described in figure 4 .

lipid head groups. RBC are exposed to varying $\mathrm{pH}$ levels in the human body depending on whether they are located in healthy tissue or in inflamed regions, were the $\mathrm{pH}$ can drop down to about $\mathrm{pH} 6$ [27]. We here study red blood cell ghosts as the absorbance of hemoglobin interferes with the Laurdan fluorescence signal. Figure $6 \mathrm{a}$ shows the temperature dependent GP of $\mathrm{RBC}$ at the $\mathrm{pH}$ levels 5,6 and 7. The GP curve shifts for $\mathrm{pH} 6$ slightly, for $\mathrm{pH} 5$ more pronounced towards higher temperatures meaning an expansion of the ordered phase due to protonation shown in the inset of figure $6 \mathrm{a}$ for a constant temperature of $\mathrm{T}=37^{\circ} \mathrm{C}$. Therefore, RBC membranes react to $\mathrm{pH}$ changes as it is known for synthetic membranes containing charged headgroups. The transition calculated by data fitting and building the derivative with respect to temperature in figure $6 \mathrm{~b}$ shows the same behavior. While there is almost no change from $\mathrm{pH} 7$ to $\mathrm{pH} 6$ the melting temperature $T_{m}$ shifts at $\mathrm{pH} 5$ by $\Delta T_{m}$ $=+1.5 \mathrm{~K}$ to the right compared to $\mathrm{pH} 7$. Cooperativity and melting enthalpy are reduced by protonation. The $\mathrm{pH}$ dependent shift in the physiological temperature regime depicted in the upper inset again can be translated in a $\triangle G P$ with corresponding equivalent temperature change $\Delta \mathrm{T}$ (Fig. 6c). For $\mathrm{pH} 7$ lipid order is increased as it would be after a temperature reduction of $\Delta T=-4 \mathrm{~K}$ to $\Delta \mathrm{T}$ $=-6 \mathrm{~K}$.

\section{Conclusions}

In summary we have not only shown the presence of broad order/disorder phase transitions in biological membranes but also that they are affected by cholesterol, anaesthetic and $\mathrm{pH}$ in the same way as it is known for sharp transitions in synthetic membranes. Even though the transition itself is not visible in the physiological temperature regime where lipid order changes only linearly with temperature, the knowledge about the underlying physics of this effect allows for prediction how membrane order reacts to environmental changes. This approach could prove itself useful since our findings that lipid order is affected by adaptation processes and morphological changes during differentiation or trypsination suggest that cellular functionality is indeed connected to membrane state. Exciting studies based on these results may include but are not limited to in-depth studies on substance and signal transport across and along the membrane. 


\section{Acknowledgements}

The authors thank Prof. Dr. Matthias

Schneider and Simon Fabiunke for fruitful

discussions. The authors thank the Center

for Nanoscience (CeNS) and the

Augsburg Centre for Innovative

Technologies (ACIT) for funding. C.W.

would like to acknowledge funding for the

project "Physical and functional interaction mechanisms at cell membranes and

vessel walls" by the University of

Augsburg. N.F. would like to thank the

Joachim Herz foundation. Furthermore,

the authors thank Dr. Stefanie Grützner for providing blood samples.

\section{Data availability}

All data that support our findings are available from the corresponding authors upon reasonable request. There are no restrictions on data availability.

\section{Author contributions}

N.F. and C.W. designed research; N.F. and C.W. performed research; N.F. analyzed data and implemented experimental tools; N.F. and C.W. wrote the paper.

\section{Competing interests}

The authors declare no competing interests.

\section{References}

[1] Blicher A, Wodzinska K, Fidorra M, Winterhalter M, Heimburg T. The temperature dependence of lipid membrane permeability, its quantized nature, and the influence of anesthetics. Biophys $\mathrm{J}$ 2009;96:4581-91. https://doi.org/10.1016/j.bpj.2009.01 .062 .

[2] Ebel H, Grabitz P, Heimburg T. Enthalpy and volume changes in lipid membranes. I: The proportionality of heat and volume changes in the lipid melting transition and its implication for the elastic constants. J Phys Chem B
2001;105:7353-60.

https://doi.org/10.1021/jp010515s.

[3] Heimburg T, Jackson AD. On

soliton propagation in

biomembranes and nerves. Proc

Natl Acad Sci U S A

2005;102:9790-5.

https://doi.org/10.1073/pnas.050382 3102.

[4] Strobl FG, Seitz F, Westerhausen

C, Reller A, Torrano AA, Bräuchle

$C$, et al. Intake of silica

nanoparticles by giant lipid vesicles:

Influence of particle size and thermodynamic membrane state.

Beilstein J Nanotechnol

2014;5:2468-78.

https://doi.org/10.3762/bjnano.5.256

[5] Parasassi T, Krasnowska EK,

Bagatolli L, Gratton E. Laurdan and

Prodan as polarity-sensitive

fluorescent membrane probes 1998;8:365-73.

[6] Scomparin C, Lecuyer S, Ferreira $\mathrm{M}$, Charitat T, Tinland B. Diffusion in supported lipid bilayers: Influence of substrate and preparation technique on the internal dynamics. Eur Phys J E 2009;28:211-20. https://doi.org/10.1140/epje/i200810407-3.

[7] Böckmann RA, Hac A, Heimburg T, Grubmüller $\mathrm{H}$. Effect of sodium chloride on a lipid bilayer. Biophys $\mathrm{J}$ 2003;85:1647-55.

https://doi.org/10.1016/S00063495(03)74594-9.

[8] Heimburg T. The capacitance and electromechanical coupling of lipid membranes close to transitions: The effect of electrostriction. Biophys J 2012;103:918-29. https://doi.org/10.1016/j.bpj.2012.07 010 .

[9] Trauble $\mathrm{H}$, Eibl H. Electrostatic effects on lipid phase transitions: membrane structure and ionic environment. Proc Natl Acad Sci U S A 1974;71:214-9. https://doi.org/10.1073/pnas.71.1.21 4. 
[10] Busto J V., García-Arribas AB, Sot J, Torrecillas A, Gómez-Fernández JC, Goñi FM, et al. Lamellar gel $(L \beta)$ phases of ternary lipid composition containing ceramide and cholesterol. Biophys $\mathrm{J}$ 2014;106:621-30.

https://doi.org/10.1016/j.bpj.2013.12 .021 .

[11] McElhaney RN. Differential scanning calorimetric studies of lipid-protein interactions in model membrane systems. BBA - Rev Biomembr 1986;864:361-421. https://doi.org/10.1016/03044157(86)90004-3.

[12] Parasassi T, Conti F, Gratton E. Time-resolved fluorescence emission spectra of Laurdan in phospholipid vesicles by multifrequency phase and modulation fluorometry. Cell Mol Biol 1986;32:103-8.

[13] Parasassi, $T$ and De Stasio, $G$ and Ravagnan, $\mathrm{G}$ and Rusch, RM and Gratton E. Quantitation of lipid phases in phospholipid vesicles by the generalized polarization of Laurdan fluorescence. Biophys $\mathrm{J}$ 1991;60:179-89.

[14] Bacalum M, Zorila B, Radu M. Fluorescence spectra decomposition by asymmetric functions: Laurdan spectrum revisited. Anal Biochem 2013;440:123-9. https://doi.org/10.1016/j.ab.2013.05. 031.

[15] Himbert S, Alsop RJ, Rose M, Hertz L, Dhaliwal A, Moran-Mirabal JM, et al. The Molecular Structure of Human Red Blood Cell Membranes from Highly Oriented, Solid Supported Multi-Lamellar Membranes. Sci Rep 2017;7:1-14. https://doi.org/10.1038/srep39661.

[16] Folch, J. and Lees, M. and Sloane Stanley GH. A simple method for the isolation and purification of total lipides from animal tissues. J Biol Chem 1957;226:497-509.

[17] Feijó Delgado F, Cermak N, Hecht
VC, Son S, Li Y, Knudsen SM, et al. Intracellular Water Exchange for Measuring the Dry Mass, Water Mass and Changes in Chemical Composition of Living Cells. PLoS One 2013;8.

https://doi.org/10.1371/journal.pone. 0067590.

[18] Barton PG., Gunstone FD.

Hydrocarbon chain packing and molecular motion in phospholipid bilayers formed from unsaturated lecithins. J Biol Chem 1975;250:4470-6.

[19] Davis PJ, Keough KMW. Differential Scanning Calorimetric Studies of Aqueous Dispersions of Mixtures of Cholesterol with Some Mixed-Acid and Single-Acid

Phosphatidylcholines. Biochemistry 1983;22:6334-40. https://doi.org/10.1021/bi00295a045

[20] Lewis RN, Sykes BD, McElhaney RN. Thermotropic Phase Behavior of Model Membranes Composed of Phosphatidylcholines Containing Cis-Monounsaturated Acyl Chain Homologues of Oleic Acid: Differential Scanning Calorimetric and31P NMR Spectroscopic Studies. Biochemistry 1988;27:8807. https://doi.org/10.1021/bi00403a007

[21] Huang Z, London E. Cholesterol lipids and cholesterol-containing lipid rafts in bacteria. Chem Phys Lipids 2016;199:11-6. https://doi.org/10.1016/j.chemphysli p.2016.03.002.

[22] Mužić T, Tounsi F, Madsen SB, Pollakowski D, Konrad M, Heimburg $\mathrm{T}$. Melting transitions in biomembranes. Biochim Biophys Acta - Biomembr 2019;1861:183026. https://doi.org/10.1016/j.bbamem.20 19.07.014.

[23] Fichtl B, Shrivastava S, Schneider MF. Protons at the speed of sound: Predicting specific biological 
signaling from physics. Sci Rep

2016;6:1-9.

https://doi.org/10.1038/srep22874.

[24] Fichtl B, Silman I, Schneider MF.

On the Physical Basis of Biological

Signaling by Interface Pulses.

Langmuir 2018;34:4914-9.

https://doi.org/10.1021/acs.langmuir .7b01613.

[25] Heimburg T, Jackson AD. The thermodynamics of general anesthesia. Biophys $\mathrm{J}$ 2007;92:3159-65.

https://doi.org/10.1529/biophysj.106 .099754.

[26] Labotka RJ, Omachi A. The pH dependence of red cell membrane transport of titratable anions studied by NMR spectroscopy. J Biol Chem 1988;263:1166-73.

https://doi.org/10.1016/s00219258(19)57281-7.

[27] Buchegger S, Kamenac A, Fuchs S, Herrmann R, Houdek P, Gorzelanny $C$, et al. Smart antimicrobial efficacy employing $\mathrm{pH}$-sensitive $\mathrm{ZnO}$-doped diamond-like carbon coatings. Sci Rep 2019;9:1-10. https://doi.org/10.1038/s41598-01953521-7. 


\section{Figures}
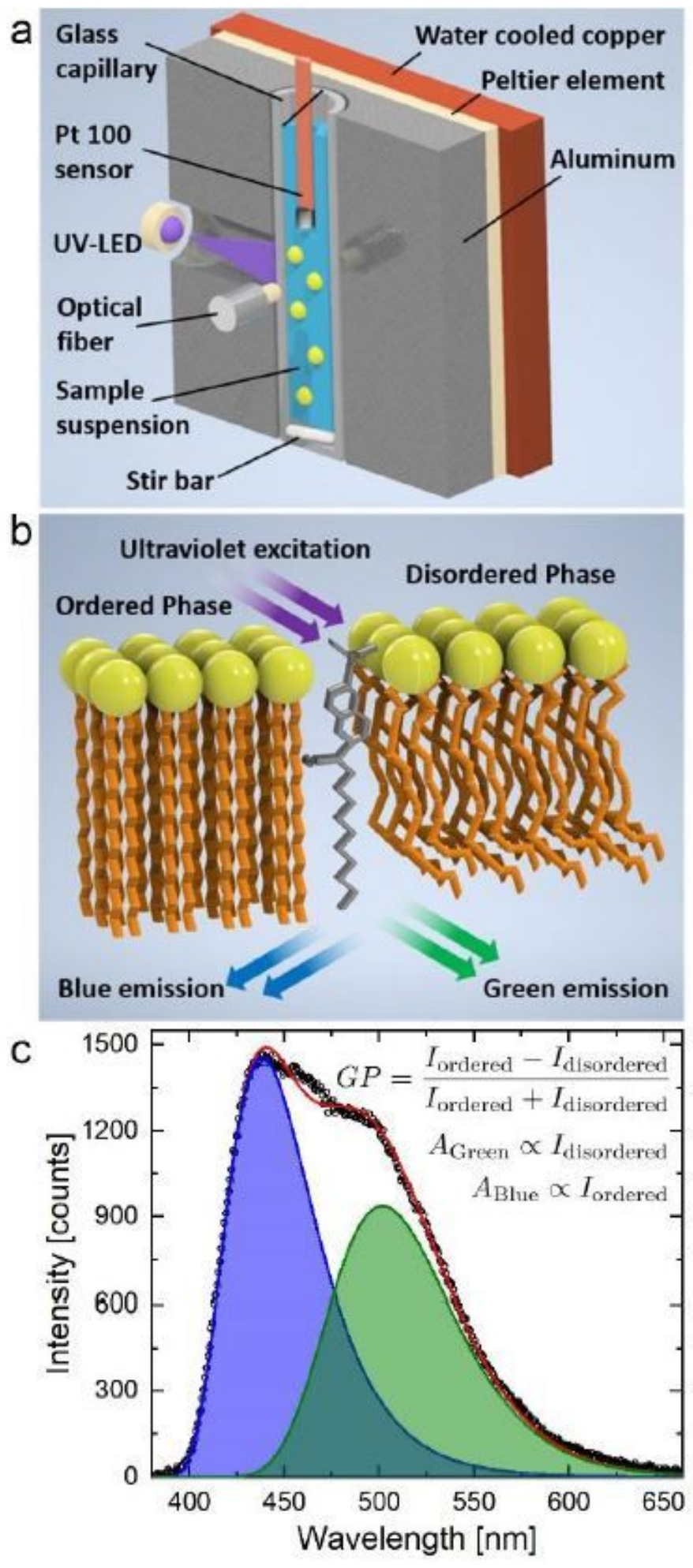

Figure 1

Experimental setup and data analysis. a For optical analysis the sample suspension is placed in a glass capillary that can be temperature controlled in a broad temperature range. The fluorescence emission is recorded by a spectrometer attached to an optical fiber. $\mathrm{b}$ The membrane embedded dye Laurdan allows 
for determination of the phase state of lipid membrane samples due to its polarity sensitive emission spectrum. c The fluorescence intensities originating from ordered/disordered lipid domains are determined by data fitting to calculate the generalized polarization as measure for the membrane phase state.
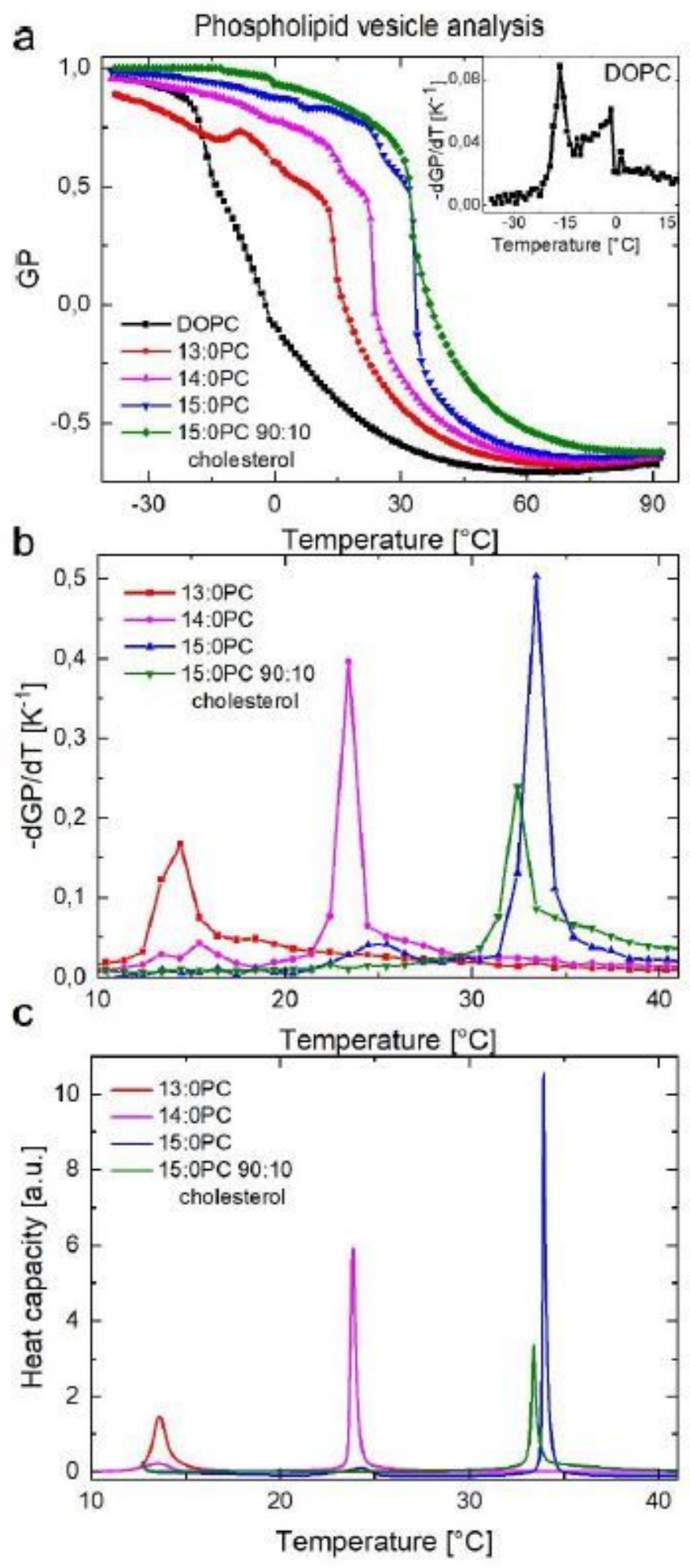

Figure 2 
Phospholipid vesicle analysis. a Temperature dependent generalized polarization of various phospholipid multilamellar vesicles measured during heating. The inset shows the derivative with respect to temperature of DOPC vesicles with a peak at $T=-16.5^{\circ} \mathrm{C}$ indicating the ordered/disordered phase transition. b Derivative with respect to temperature of various lipid vesicles showing the same ordered/disordered phase transitions as their heat capacity profiles measured by DSC in c.


Figure 3 
Comparison of phospholipid vesicles and HeLa cell membranes. a, b Normalized emission spectra of 14:OPC multilamellar vesicles/HeLa cells as a function of temperature and fluoresccnce microscopy images of the samples (field of view about $420 \times 320 \mu \mathrm{m}^{2}$ ). c GP of 14:OPC multilamellar vesicles/HeLa cells as a function of temperature and their corresponding derivatives after temperature at two differently scaled axes.
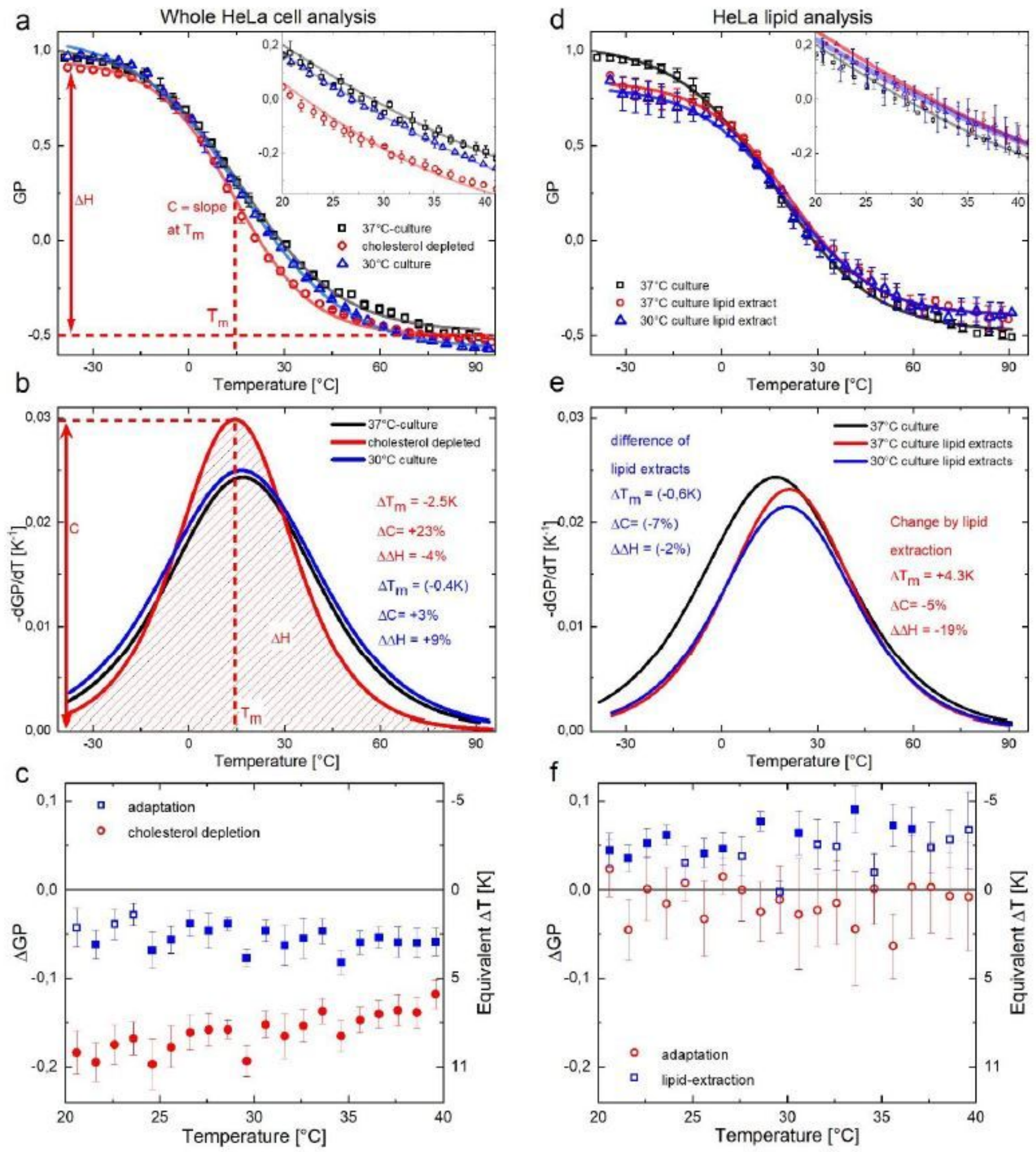

Figure 4 
Phase state analysis of HeLa cell membranes and their lipid extracts under varying conditions. a,d Temperature dependent GP values of untreated, cholesterol depleted and low temperature $\left(30^{\circ} \mathrm{C}\right)$ adapted HeLa cell membranes and their lipid extracts. Each data point represents the mean value with standard deviation of at least 3 independent samples. For visibility only every fourth point is shown. The data is approximated by a Boltzmann sigmoid function which allows for determination of the point of inflection or $\mathrm{Tm}$, its steepness as a measure for cooperativity $\mathrm{C}$ and the span corresponding to a melting enthalpy $\Delta \mathrm{H}$. The inset shows GP values in a physiologically relevant temperature interval and the $95 \%$ confidence intervals of the according fit functions. b,e Derivatives after temperature of GP graphs pictured in A and D. The changes of melting temperature $\Delta \mathrm{Tm}$, cooperativity $\Delta \mathrm{C}$ and melting enthalpy $\Delta \Delta \mathrm{H}$ due to adaptation, cholesterol depletion and lipid extraction are calculated by comparison of the Boltzmann sigmoid fit parameters. c,f Temperature dependent GP changes induced by adaptation, cholesterol depletion and lipid extraction. Data points with a P-value larger than $5 \%$ are represented by hollow symbols. The equivalent temperature changes are calculated by the use of a linear approximation of untreated HeLa cells in the temperature regime from $20^{\circ} \mathrm{C}$ to $40^{\circ} \mathrm{C}$. 

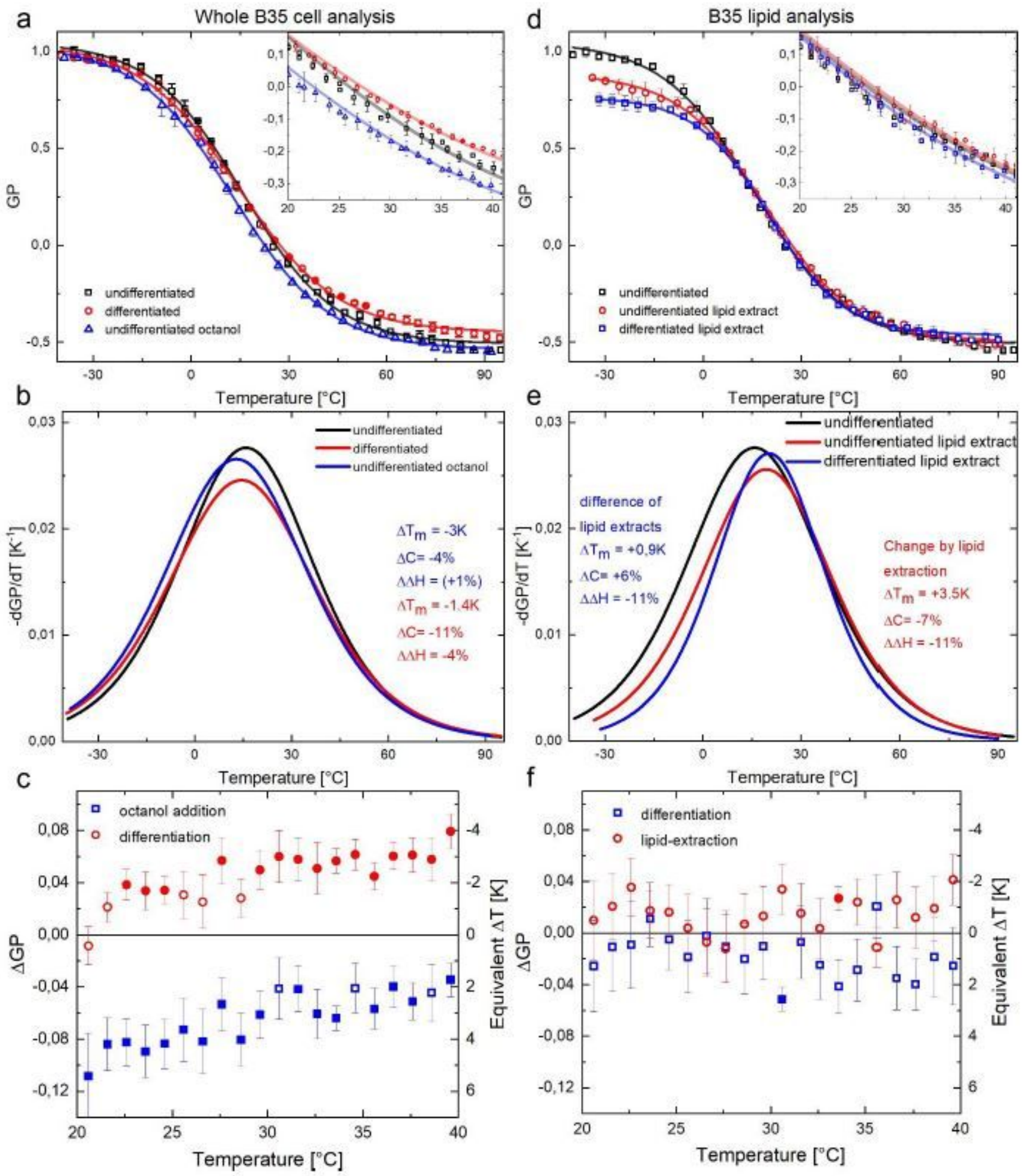

\section{Figure 5}

Phase state analysis of B35 Neuroblastoma cell membranes and their lipid extracts under varying conditions. a,d Temperature dependent GP values of untreated, differentiated and octanol (1mM) exposed cell membranes and their lipid extracts. Data point representation, error bars and fit values in a-f are treated as described in figure 4. 

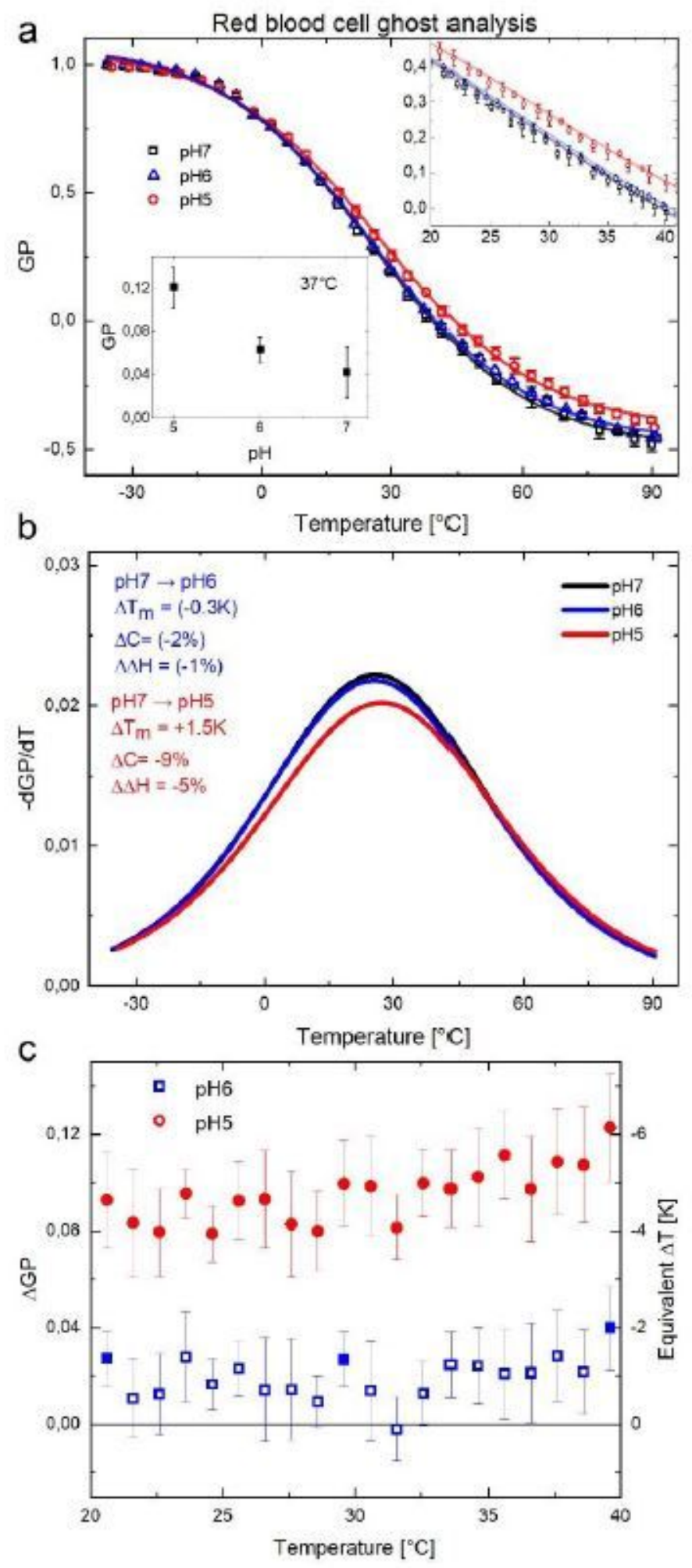

\section{Figure 6}

Phase state analysis of red blood cell ghosts under varying $\mathrm{pH}$ levels. a Temperature dependent GP values of red blood cell ghosts at $\mathrm{pH} 7, \mathrm{pH} 6$ and $\mathrm{pH} 5$. Data point representation, error bars and fit values in a-c are treated as described in figure 4.

\section{Supplementary Files}


This is a list of supplementary files associated with this preprint. Click to download.

- SupplementaryInformationtoFaerber2021.pdf

- SupplementaryInformationtoFaerber2021.pdf 